\title{
An Agarose-Gel Based Method for Transporting Cell Lines
}

\author{
Lingzhi Yang ${ }^{1,2}$, Chufang $\mathrm{Li}^{1}$, Ling Chen ${ }^{1,2}$ and Zhiyuan $\mathrm{Li}^{*}, 1,2$ \\ ${ }^{1}$ State Key Laboratory of Respiratory Diseases, Guangzhou Institute of Biomedicine and Health, Chinese Academy of \\ Sciences (GIBH), Guangzhou 510663, China \\ ${ }^{2}$ College of Life Science, University of Science and Technology of China (USTC), Hefei 230027, China
}

\begin{abstract}
Cryopreserved cells stored in dry ice or liquid nitrogen is the classical method for transporting cells between research laboratories in different cities around the world in order to maintain cell viability. An alternative method is to ship the live cells in flasks filled with cell culture medium. Both methods have limitations of either a requirement on special shipping container or short times for the cells to survive on the shipping process. We have recently developed an agarose gel based method for directly transporting the live adherent cells in cell culture plates or dishes in ambient temperature. This convenient method simplifies the transportation of live cells in long distance that can maintain cells in good viability for several days.
\end{abstract}

Keywords: Cell transportation, cell shipment, live cell shipment, agarose gel, agarose gel based cell preservation, cryopreserved cells.

\section{INTRODUCTION}

Cell lines have been extensively used in research laboratories both in academia and industry that are useful tools for basic research and drug discovery. Cell lines are available from different sources including commercial supplies, and laboratories in both academia and industry. It is a common practice to transport cells from a city to another city as well as from a country to another country. High viability of received cells after thawing is an imminent requirement for the cell transportation.

Cryopreserved cells in dry ice and live cells in complete medium are commonly used for cell shipments. Cryopreservation is classical way to store cells that doesn't significantly affect cell properties demonstrated by cell fractionation studies and biochemical analysis [1]. It was reported that the peripheral bloods mononuclear cells had better viral antigen proliferation responses preserved in cryopreservation compared to specimens shipped overnight in complete medium [2]. However, the process of cryopreservation of cells requires multiple steps in three days with a special container and liquid nitrogen to keep ultra-low temperature. The shipping of cryopreserved cells requires a dry ice container for next day delivery that is inconvenient and costly. During the shipment, it may encounter risks such as formation of intracellular ice crystal, osmotic and chilling injury, cytoplasm fracture or even effects on the cytoskeleton or genomic structures [3].

Alternatively, live cells in a flask filled with complete medium have been transported between laboratories [4, 5]. Typically, a tissue culture flask with cells is filled with cell culture medium to avoid shaking with air that could damage

*Address correspondence to this author at the Guangzhou Institute of Biomedicine and Health, Chinese Academy of Sciences, Guangzhou International Business Incubator, Guangzhou Science Park, 510663, China; Tel: 20-32293266; Fax: 20-32290606; E-mail: li_zhiyuan@gibh.ac.cn cells. Limited shipping time (usually up to 24 hours) is a major disadvantage for this method that is due to rapid exhaustion of oxygen and $\mathrm{pH}$ change in the medium [6]. Furthermore, shipment of liquid specimen is not convenient in air travel. Thus, an alternative convenient and long lasting method for transporting live cells should resolve many problems in cell shipment and facilitate the scientific researches using live cells.

Here we describe a complement method to transport cells coated with agarose gel in plates or dishes. Several cell lines including MDCK, HEK-293 and A549 cells have been evaluated in this method in which high cell viabilities have been observed. We have demonstrated that this method can keep cell viable for several days at ambient temperature.

\section{MATERIALS AND METHODS}

\section{Cell Lines and Medium}

The Madin Darby Canine Kidney (MDCK), Human Embryonic Kidney 293 (HEK-293), Human lung adenocarcinoma epithelial (A549) cell lines were used throughout this study. The medium for cell maintenance was composed of DMEM (Dulbecco's Modified Eagle's Medium) supplemented with $10 \%$ FBS (Fetal Bovine Serum). $2 \times$ MEM was purchased from Invitrogen/GIBCO and Agarose was Biowest-regular (Gene Tech, Shanghai, China).

\section{Cell Culture}

Cells were maintained in tissue culture flasks in complete medium at $37{ }^{\circ} \mathrm{C}$ in a humidified incubator supplied with 5\% $\mathrm{CO}_{2}$. For following experiments, cells were seeded at $1 \times 10^{5}$ cells per well in a 12-well plate with $1 \mathrm{ml}$ medium/well and incubated for $24 \mathrm{hrs}$ at $37{ }^{\circ} \mathrm{C}$ in a humidified incubator supplied with $5 \% \mathrm{CO}_{2}$.

\section{Preparation of Agarose-Medium Solution}

Agarose (catalog \#: 111-760) was purchased from Gene Tech Company (Shanghai, China). A 2x agarose solution 
was made in $\mathrm{dH}_{2} \mathrm{O}$, melted by heating and warmed at $56{ }^{\circ} \mathrm{C}$ in a water bath. The $2 \mathrm{x}$ agrose solution was then mixed with an equal volume of $2 \mathrm{x}$ medium (with $10 \% \mathrm{FBS}$ ) to form a $1 \%$ agarose-medium mixture and kept at $45{ }^{\circ} \mathrm{C}$ in a water bath before use.

\section{Preparation of Cells for the Shipment}

After overnight culture, the medium was aspirated out of the cell plate and $500 \mu \mathrm{l} /$ well $1 \%$ agrose-medium at $45{ }^{\circ} \mathrm{C}$ was added to the plate. The plate was covered with a lid and kept at room temperature until agarose became solid. The edges of the 12-well plate were then sealed with parafilm (Whatman) and the plate was ready for shipment.

\section{Recovery of Cells After Transportation}

Upon receiving the cells from shipment, the parafilm seal was removed from the cell plate. The agarose-medium gel was lifted gently with forceps and discarded. The cell culture medium was added to the cell plate immediately and the plate was then incubated in a $37{ }^{\circ} \mathrm{C}$ humidified incubator supplied with $5 \% \mathrm{CO}_{2}$ for 1 to 3 days.

\section{Cell Viability Test}

The cell viability was measured by the counting the cell numbers in a hemocytometer using the trypan blue exclusion assay. After recovered from transportation (usually 1-2 days), cells were trypsinized and resuspended in medium. A 1:1 volume mixing of $0.1 \mathrm{ml}$ cell suspension and trypan blue reagent $(0.4 \%$, Invitrogen) was prepared for cell counting. The numbers of live cells and dead cells were counted with the trypan blue staining method. To ensure the reproducibility of this cell transportation method, a total of 4 independent experiments were carried out for experimental each condition.

\section{Colony Formation Assay}

After discarding agarose-medium gel, cells were cultured in complete medium at $37{ }^{\circ} \mathrm{C}$ for $24 \mathrm{hrs}$ to recover. The recovered cells were then trypsinized and resuspended in medium. For the colony formation experiment, 500 cells were seeded in a $6 \mathrm{~cm}$ dish and cultured for 7 to 10 days in a $37^{\circ} \mathrm{C}$ humidified incubator supplied with $5 \% \mathrm{CO}_{2}$. The medium was changed every 3 days to supply cells with fresh nutrition. When the cell clone grew visible, the medium was removed and the cell was fixed with $10 \%$ formalin for $10 \mathrm{~min}$ utes. Then the dish was washed with PBS for three times and the cell colony was stained with $0.2 \%$ crystal violet (in $10 \%$ formalin ) for 15 minutes. The numbers of cell colonies were counted after wash.

\section{RESULTS AND DISCUSSION}

\section{Agarose Concentration and Cell Viability}

Agarose was widely used in viral plaque assays at a concentration of $\sim 0.8 \%$. For the purpose of cell transportation, the agarose-medium gel should have enough rigidity to support adherent cells and prevent cell damage in addition to supplements of the nutrition and moisture to cells. In this experiment, a series of agarose concentrations $(0.5,0.8,1$, 1.2 and $1.5 \%$ ) were tested for the agorose-medium gel to cover the MDCK cells after one-day culture ( $\sim 60 \%$ confluence). After three days at room temperature to mimic shipping condition, the cells were recovered in culture as the steps described in Method. We found that $1.0 \%$ agarose was optimal concentration for the agarose-medium gel covered cells that were kept at room temperature for 1 to 3 days to mimic transportation (Figs. 1A, B). The cells were recovered with the higher viability at this agarose concentration. We also found that cell recovery viabilities were similar after the cells were transported in a plate covered with a layer of aga-
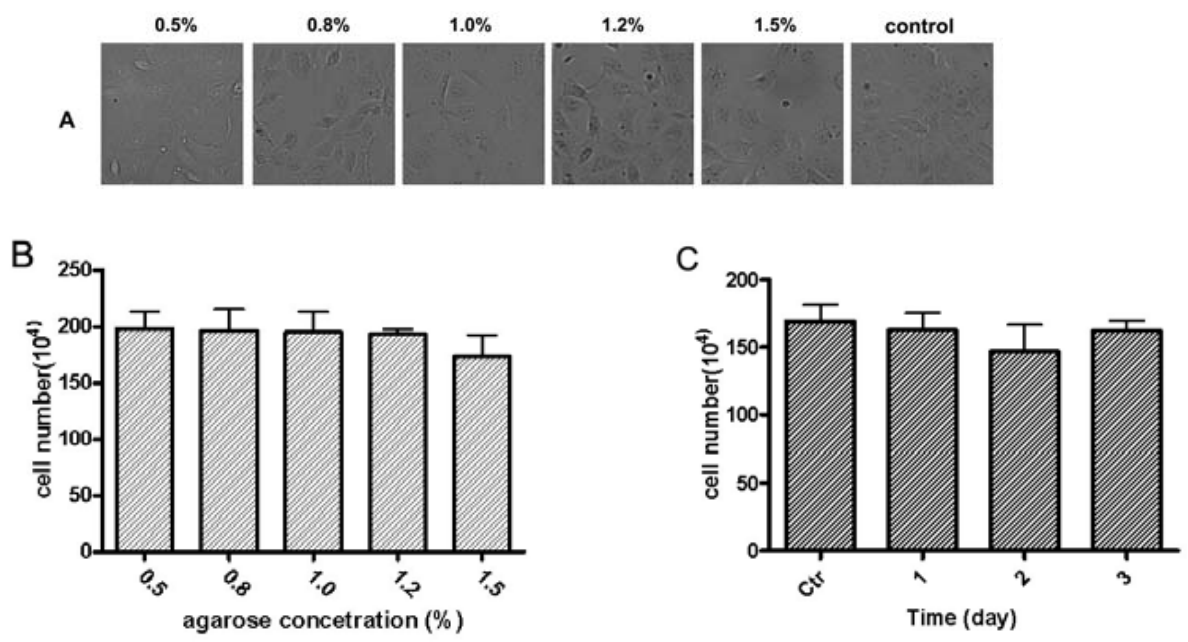

Fig. (1). Comparison of cell growth rate in a plate covered with a layer of agarose-medium gel with the control cells which were maintained in the regular cell culture condition. (A) Images of the MDCK cells that were recovered and incubated for 24 hours in the regular cell culture condition after the three days transporting in the agarose-medium gel covered plate. The agarose concentrations in the gel were $0.5,0.8,1$, 1.2 and $1.5 \%$, respectively. The control cells were kept in the regular cell culture condition. (B) Comparison of effect of agarose concentrations on the recovered cell numbers. The cells were seeded and cultured one day at $37{ }^{\circ} \mathrm{C}, 5 \% \mathrm{CO} 2$ incubator before covered with the agarose-medium gel with different agarose concentrations for transportation. After three days of transportation, the gel was peeled and regular cell culture medium was added to cells. The cell numbers of the MDCK cells were counted after the cells were cultured in the regulate cell culture condition for one day. (C) Comparison of recovered cell numbers after transported in 1\% agarose-medium gel for 1,2 or 3 days at ambient condition. 


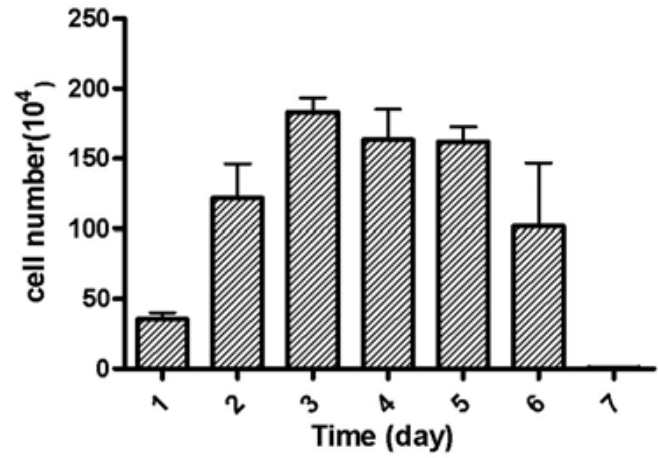

Fig. (2). The numbers of recovered MDCK cells coated with $1 \%$ agarose. $0.4 \times 10^{6}$ MDCK cells were seeded in $60 \mathrm{~mm}$ dish to grow for 24 hours at $37{ }^{\circ} \mathrm{C}, 5 \% \mathrm{CO} 2$ incubator, and then MDCK cells were coated with $1 \%$ agarose-medium and mimic shipping condition for 1 to 7 days. The recovered cells were counted after removing the agarose-medium gel and a 24 hours incubation in the regular cell culture condition.

rose-medium gel for 1 to 3 days (Fig. 1C). The agarosemedium gel layer provides cells with the proper moisture and nutrition to maintain cell alive with a slow growth rate during the shipment. It also provides an ideal cushion support for cells during transportation.

We have studied the relationship of cell numbers with the days after the cells were preserved in the agarose-medium gel condition. The agarose-medium gel layer was removed on 1-6 days after transportation by directly peeling the layer off the cell plate. The MDCK cells covered with $1 \%$ agarose-medium gel grew slowly in the first three days which reached a plateau between 3-5 days (Fig. 2). The cell number started reducing at the six day, indicating that this is maximal transportation time for this method. We found the mor- phology of the cells after recover was same as the normal cell culture condition (data not shown). The results indicated that the MDCK cell covered with the agarose-medium gel were healthy up to five days in ambient condition and this method for long distance transport of live cells.

We also found that the directly peeling of the agarosemedium gel layer could cause the partial loss of cells because the agarose gel could take some cells with it. An alternative method is to liquefy agoarose gel by agarase followed by aspiration of liquefied medium out of wells as it was reported [7]. This method for removing agarose gel layer should reduce the partial loss of cells due to directly peeling of the agarose gel.

For the cell transportation using this agarose-medium gel method, the temperature should be between 1 to $37^{\circ} \mathrm{C}$ since great than $37{ }^{\circ} \mathrm{C}$ or lower than $0{ }^{\circ} \mathrm{C}$ can damage the cells. The agarose layer is not hard enough at concentration less than $0.8 \%$ and becomes dry and easy to crack above $1.5 \%$. Thus, the agarose concentrations from 0.8 to $1.2 \%$ should be used for cell transportation.

\section{Effect of Agarose-Medium Gel on Colony Formation}

We used this colony formation experiment to test the viability and function of the agarose-medium gel preserved cells after transportation. In this colony formation experiment, cells are sparsely seeded in $6 \mathrm{~cm}$ dish (e.g. 500 cells/dish) and cultured for 7 to 10 days for the colonies to grow. The numbers of colony formation in the recovered cells after being kept 1 to 3 days in the agarose-medium gel at room temperature were similar to these in regular cell culture condition. The results of colony formation experiment showed that the morphology and size of cell colonies for three cell lines were remained similar after recovering from the transportation with the agarose-medium gel method (Fig. 3). We observed that the number of colonies was reduced in the HEK293 and A549 cell lines after the 1.2\% agarose

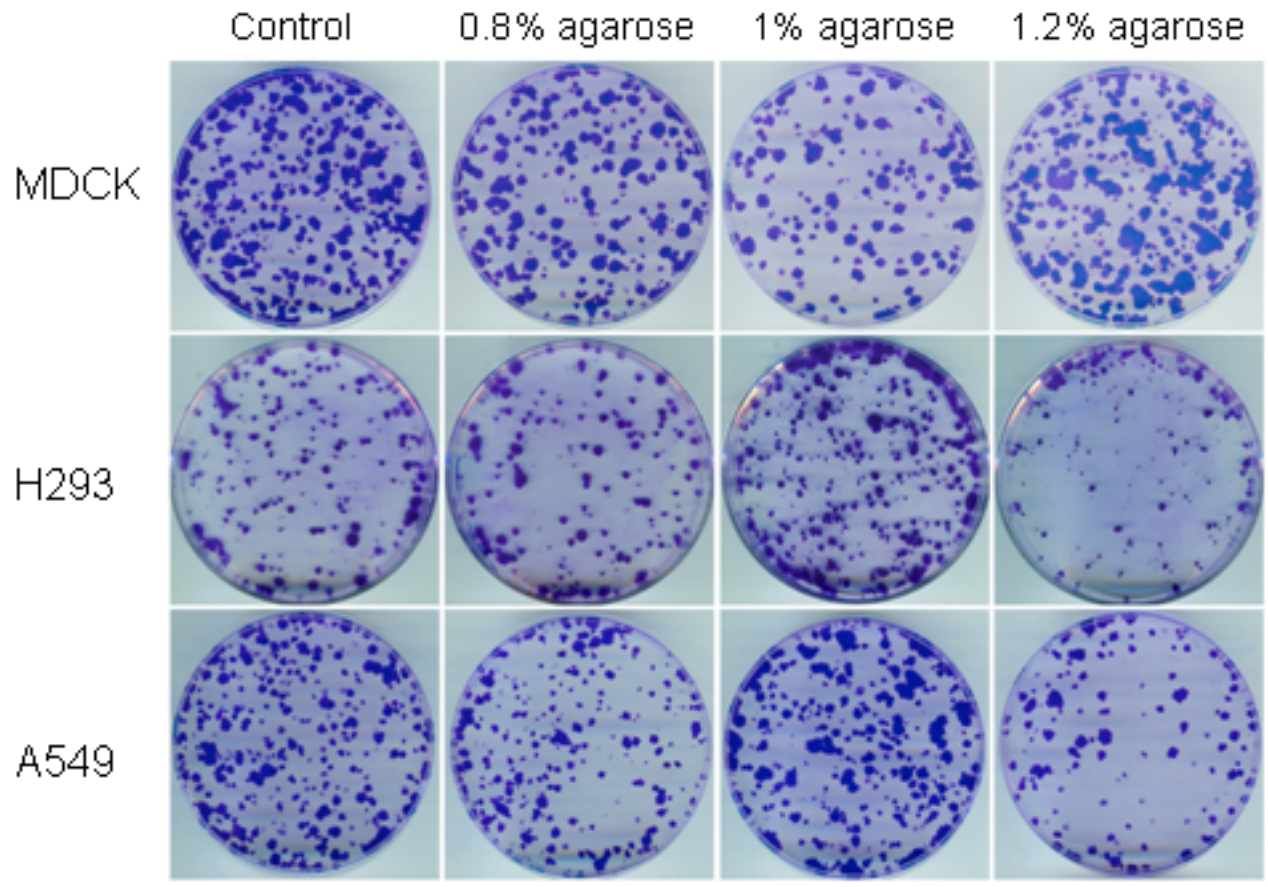

Fig. (3). The Effect of agarose concentration on colony-formation. 
treatment though these remained unchanged at the lower agarose concentration (Fig. 3). This result suggested that the agarose concentration higher than $1.2 \%$ might may reduce the cell viability with this transportation method. Thus, agarose concentration at $1 \%$ is an optimal condition for this cell transportation method because it has the highest cell recovery viability and is firm enough to form a proper layer of gel to protect cell during the transportation.

Traditional cell shipping methods need a special container and dry ice or need to fill flasks of live cells with medium. In comparison, this agarose-medium gel based method for transporting live cells directly in a plate (e.g. 6-well plate) has advantages of simple, low cost, no requirements for special container and dry ice, and no liquid in cell plate. Cells shipped in live condition can be recovered quickly upon receiving. The recovery rate of cells using this method is also acceptable compared with the traditional methods. Therefore, this agarose-medium gel based method is useful and convenient way for transporting cells between cities and countries. We recommend that the time for cells transporting in this method should be in 3 days (no more than 5 days) after the cells are covered with the agarose-medium gel.

\section{ABBREVIATIONS}

DMEM $=$ Dulbecco's Modified Eagle's Medium

FBS = Fetal Bovine Serum, PS: penicillin- streptomycin

\section{ACKNOWLEDGEMENTS}

The authors thank Dr. Pan Weiqi for her technique discussion.

\section{REFRENCES}

[1] Prento P. The effects of freezing, storage, and thawing on cell compartment integrity and ultrastructure. Histochem Cell Biol 1997; 108: 543-7.

[2] Weinberg A, Betensky RA, Zhang L, Ray G. Effect of shipment, storage, anticoagulant, and cell separation on lymphocyte proliferation assays for human immunodeficiency virus-infected patients. Clin Diagn Lab Immunol 1998; 5: 804-7.

[3] Dong Q, Huang C, Eudeline B, Tiersch TR. Systematic factor optimization for cryopreservation of shipped sperm samples of diploid Pacific Oysters, Crassostrea gigas. Cryobiology 2005; 51: 17697.

[4] Coriell LL. Preservation, storage, and shipment. Methods Enzymol 1979; 58: 29-36.

[5] Broomfield A, Bourn D. Basic techniques in molecular genetics. J Laryngol Otol 1998; 112: 230-4.

[6] Betensky RA, Connick E, Devers J, et al. Shipment impairs lymphocyte proliferative responses to microbial antigens. Clin Diagn Lab Immunol 2000; 7: 759-63.

[7] Carlssson J, Malmqvist M. Effects of bacterial agarase on agarose gel in cell culture. In Vitro 1997; $7: 417-22$.

Received: May 05, 2009

(C) Yang et al.; Licensee Bentham Open.

This is an open access article licensed under the terms of the Creative Commons Attribution Non-Commercial License (http://creativecommons.org/licenses/by-nc/3.0/) which permits unrestricted, non-commercial use, distribution and reproduction in any medium, provided the work is properly cited. 